\title{
Space-Time-Frequency Tight Coupling Routing Algorithm Based on Delay-Tolerant Pheromone Dispersion Search
}

\author{
Ning $\mathrm{Li}^{1}$, Run-Tao Zhu ${ }^{1} *$ and Zhong-Liang Deng ${ }^{1}$ \\ ${ }^{1}$ School of Electronic Engineering, Beijing University of Posts and \\ Telecommunications. Beijing, 100876, China \\ Inmmdsy@sina.com, rruntaozhu@163.com, 32dengzhL@bupt.edu.cn
}

\begin{abstract}
A kind of Space-Time-Frequency Tight Coupling Routing Algorithm based on DelayTolerant Pheromone Dispersion Search is proposed according to the problem of the uneven distribution of traffic according to the high dynamic and low ability of the processing capacity of the Satellite Network Service. In this method, difference of time, space, frequency domain among various satellite business are adopted to make quantitative description and dynamic learning towards the phernmone mentioned in the algorithm, thus the link status in the satellite network can be reflected by the phernmone dynamically. The new strategy can not only overcome the existing disadvantage of single reference value and low learning ability of algorithm, but also solve the local tidal effects issue resulted from the high dynamic of network and low processing capacity of satellite nodes. This algorithm can monitor the distribution of the traffic in real time and adjust the routing strategy in accordance with the results. Simulator shows that compared to the existing schemes, the algorithm can provide larger throughput and smaller time delay under the guarantee of the low packet drop rate.
\end{abstract}

Keywords: communication ; load balancing ; Delay-Tolerant Pheromone ; LEO network

\section{Introduction}

With the development of on-board processing technology and the promotion of the storage capacity, satellite communication has increasingly become a crucial part of the global information [1-3]. Compared with the ground communication network, satellite communication network has the advantages of wide coverage, multiple transmission, little influence of terrain and surface features and little dependence on existing communication in existence. However, the network also has the characteristic of high dynamic and limited processing ability. These particularities lead to the non-uniform distribution phenomenon of traffic in the network and directly trigger the reduction of throughput and increment of traffic transmission delay. The critical issue restricts the development of satellite communication. According to the statistics, present routing algorithms can only guarantee $60 \%$ of the satellite network's total utilization which obviously indicated that routing algorithm possesses highly potential to be improved. Load balancing is essential to the network traffic management. It can spread the local traffic to the spare part in order to avoiding the tidal effects. Therefore, effective network traffic load balancing which can increase the throughput and reduce the end-to-end time delay will provide great help to the promotion of the whole network's performance.

A multitude of related scholars have carried out quite a few research regarding the traffic load balancing method in satellite network. The LEO network in literature [4-6] don't consider the delay-tolerance of network fully although they are all optimized and have promotion in routing hoop and utilization. The so-called double layer satellite network routing algorithm has been investigated in [7-9]. Among them, the Explicit Load 
Balancing Routing Protocol(ELB)[7] which is applied to the node is a typical algorithm. The algorithm gives notice to the neighbor node. When the link congestion occurs in the node, it will choose suboptimal path to avoid the data sending to the congestion link. Literature [8] puts forward a novel routing algorithm based on topological variation on the basis of the dynamic of network. Literature [9] employs link perception algorithm to perceive the link status information and make routing decision. Nevertheless, the above algorithm is all incapable of integrating topological variation and uneven distribution of ground traffic together, which make the total utilization of network increase obviously.

To solve the existed problems and shortcomings, Space-Time-Frequency Tight Coupling Routing Algorithm based on Delay-Tolerant Pheromone Dispersion Search(STC-RA) is proposed. This algorithm can provide different load balancing strategy for diversified traffic accurately, which can overcome the high dynamic of satellite network effectively. The algorithm can improve average throughput of the system, reduce PDR and shorten processing time delay at the same time, thus provide a new idea to solve current load balancing problem in satellite system.

\section{Network Modeling and Problem Description}

As Multi-Layered Satellite Network (MLSN) has the advantage of high efficiency and effectiveness in data transmission, it acquires an increasing number of application. Because of the relatively static of the GEO satellite, the transmission of key information will be more reliable, which greatly increased the anti-destroying ability and robustness of satellite transmission routing. Therefore, this article will introduce the GEO - LEO double layer satellite network model. As shown in figure 1. Most of the actual ant colony algorithms study mainly focus on single layer LEO network. All traffic transmits through single network as it fail to classify information effectively, which will pose a heavy burden to the network. Compared to the LEO satellite, GEO satellite has lower routing complexity and higher stability, though it has no advantage in delay time. Consequently, if failure happens when routing, there's another choice to transfer part of the information to another network layer, which will greatly improve the reliability of network, reduce LEO communication pressure and increase whole throughput of the network indirectly.

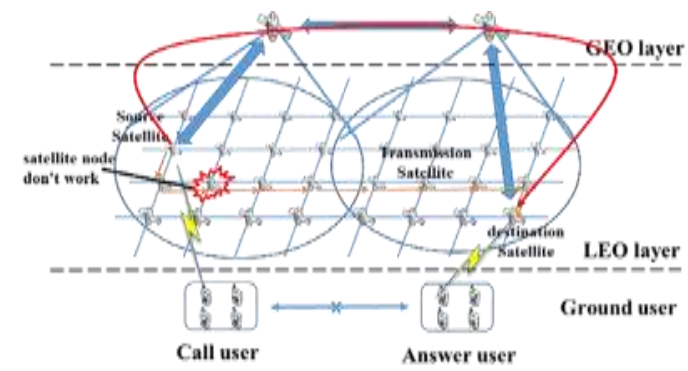

Figure 1. GEO-LEO Double Layer Satellite Network

\section{Space-Time-Frequency Tight Coupling Routing Algorithm based on Delay-Tolerant Pheromone Dispersion Search}

Learning from the idea of ant colony optimization, STC-RA is inspired by the foraging behavior of ant, which is aimed to solve optimization problem and distribution problem. The algorithm collects phernmone by creating 'ants' moved constantly around satellite network nodes. The nucleus of the algorithm is to couple distance, location and bandwidth which have a decisive role on the process of the information transmission and measure quantificationally according to concentration of phernmone. The algorithm divides the phernmone non-linear in order to let the phernmone mirror intersatellite real time link status more accurately. The 'ant' selects address of next hoop in probability in any node 
of the satellite until it arrives at destination, after which it creates backward 'ant' and goes back along the original way. This process can be represented by figure 2 .

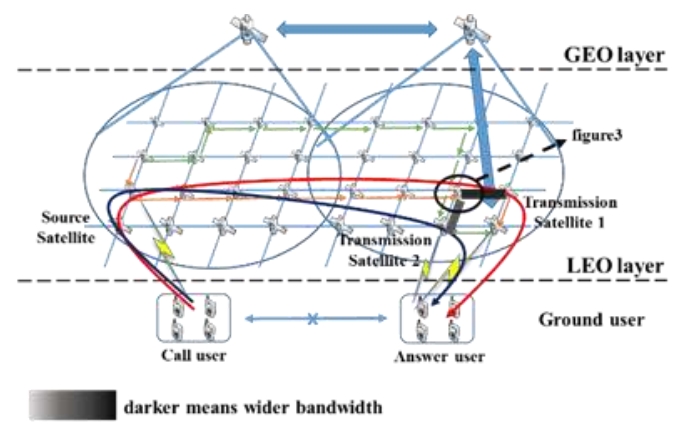

Figure 2. The Processing of Selecting Node

With the movement of 'ant' in the network, it collects 'information' and then releases, that is usedd to be the basis of other ant's selecting routing. By this method, the link has more phernmone will accumulate more 'ants' to select it and then release more phernmone, forming positive feedback effect. As shown in figure 3.

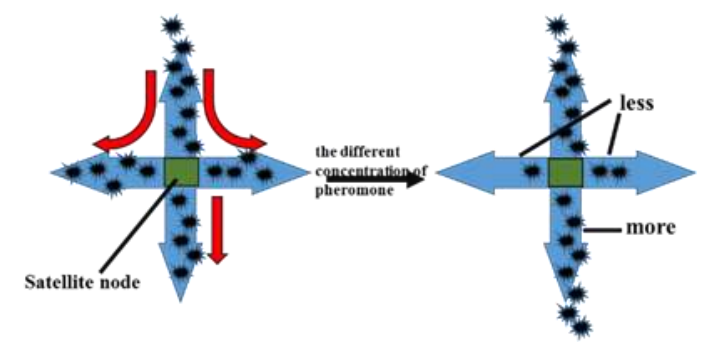

Figure 3. Gather of Ant in Satellite Node

\subsection{Parameter Definition in New Algorithm}

1)

\section{2) Definition 1: Phernmone}

In the ant colony algorithm, the pheromone is a vital parameter to describe the state of the link. According to the set of the service types[10] in this paper and diverse routing requirements of different service types, the phernmone $\tau_{s d}$ is divided into two parts, respectively for delay phernmone $\tau_{s d_{D}}$ and bandwidth phernmone $\tau_{s d_{B}}$. Delay pheromone is applied to describe the transmission time measurement between nodes in satellite and bandwidth is measured by the output buffer queue. Due to longer time delay it has, the less suitable it is for transmitting traffic. Conversely, the link has bigger weight. In consequence, it's defined as follows:

$$
\begin{aligned}
\tau_{s d_{D}} & =\lambda \log \frac{1}{\text { pDelayCost }} \\
\tau_{s d_{B}} & =\lambda \log \frac{1}{q \text { DelayCost }}
\end{aligned}
$$

Among them, pDelayCost and qDelayCost correspond to the time delay sensitive information and bandwidth sensitive information separately.

Propagation delay pDelayCost is used to express changing condition of satellite link length and interconnection relations of satellite between different orbit plane. Suppose there exists inter-satellite links between source satellite $s$ and destination satellite $d$, the propagation delay pDelayCost can be calculated by the following equation: 


$$
\text { pDelayCost }=\left\{\begin{array}{cl}
\frac{D I S T_{s-d}}{c} & \exists I S L_{s-d} \\
\infty & \text { other }
\end{array}\right.
$$

Where $D I S T_{s-d}$ donates the linear distance between satellite $s$ and satellite $d$. $C$ donates speed of light. If there is no link between satellite $s$ and satellite $d$, the delay will be set infinity.

The output buffer queue delay qDelayCost used to describe the load status of the network. The more crowded the network is, the longer the output buffer queue is and the longer time is needed. On the other hand, for bandwidth sensitive traffic, it needs more free network because of its bigger data size. The index forgetting function EFF is introduced here to demonstrate flow changes. We assumed that packet interval and the time needed for the output are both obey exponential distribution. The queue delay can be computed by M/M/1 model. Firstly, the queue packet length can be calculated by following equation:

$$
N_{\text {quewe }}=\frac{\mu_{I S L}}{1-\mu_{I S L}}
$$

Where $\mu_{I S L}$ donates average utilization. According to the forgetting index function, the utilization of ISL is: $\mu_{I S L}^{h}=l s+e^{-\Delta t_{h}} \times\left(\mu_{I S L}^{h-1}-l s\right)$.

Where $\mu_{I S L}^{h}$ and $\mu_{I S L}^{h-1}$ donate utilization. They are both calculated when the $h$ th and $h$ 1th packet arrives respectively. $e^{-\Delta t_{h}}$ is the forgetting rate and $\Delta t_{h}$ is the time interval between two arrival of two adjacent packets. $l s$ depicts the link status, that gets 0 when no data needs transmission and the queue is empty. Otherwise it gets 1 . Then we can get the output buffer queue delay as follows:

$$
q \text { DelayCost }=N_{\text {quewe }} \times \frac{P_{\text {AVG }}}{U}
$$

Where $P_{A V G}$ is average length of unit packet data and $U$ is the link bandwidth.

\section{3) Definition 2: Location Factor $\lambda$}

The parameter $\lambda$ introduced in (1) and (2) is location factors. Local hot area and nonhot area will be formed around the world due to the inhomogeneity of the global population distribution. Satellite serves for hot area will carry more communication task. We set three hot area [11] in line with the distribution of world population and traffic condition:

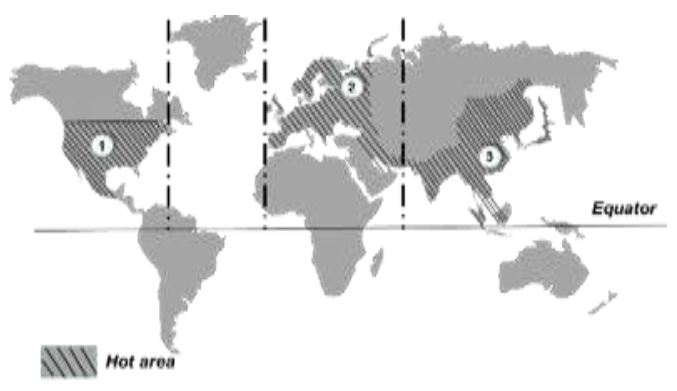

Figure 4. Distribution of Hot Area

Three hot area are divided as follows: 
Table 1. Distribution of Hot Area

\begin{tabular}{c|cccc} 
Area & \multicolumn{1}{c}{$\begin{array}{c}\text { Centre of } \\
\text { Longitude }\end{array}$} & $\begin{array}{c}\text { Covering } \\
\text { Range }\end{array}$ & $\begin{array}{c}\text { Centre of } \\
\text { Latitude }\end{array}$ & $\begin{array}{c}\text { Covering } \\
\text { Range }\end{array}$ \\
\hline $\begin{array}{c}\text { Eastern } \\
\text { Asia }\end{array}$ & $120^{\circ} \mathrm{E}$ & $60^{\circ} \mathrm{E}--180^{\circ} \mathrm{E}$ & $25^{\circ} \mathrm{N}$ & $0^{\circ}-50^{\circ} \mathrm{N}$ \\
$\begin{array}{c}\text { North } \\
\text { America }\end{array}$ & $115^{\circ} \mathrm{W}$ & $\begin{array}{c}50^{\circ} \mathrm{W}-- \\
180^{\circ} \mathrm{W}\end{array}$ & $37^{\circ} \mathrm{N}$ & $15^{\circ} \mathrm{N}--50^{\circ} \mathrm{N}$ \\
Eurasian & $5^{\circ} \mathrm{E}$ & $50^{\circ} \mathrm{W}--60^{\circ} \mathrm{E}$ & $50^{\circ} \mathrm{N}$ & $30^{\circ} \mathrm{N}--70^{\circ} \mathrm{N}$
\end{tabular}

On this basis, the hot area centre is made as the reference point and define the geographic information as follows:

$$
\lambda=e^{\frac{1}{90}\left(r_{1}-\sqrt{\left(x-r_{2}\right)^{2}+\left(y-r_{1}\right)^{2}}\right)}
$$

Where $r_{1}$ and $r_{2}$ represent the longitude and latitude of centre point. $\left(r_{1}, r_{2}\right)$ get corresponding values according to distinct range of longitude. When $50^{\circ} \mathrm{W} \leq \mathrm{y} \leq 60^{\circ} \mathrm{E}$, $\left(r_{1}, r_{2}\right)=\left(5^{\circ} \mathrm{E}, 50^{\circ} \mathrm{N}\right)$. When $180^{\circ} \mathrm{W} \leq \mathrm{y} \leq 50^{\circ} \mathrm{W},\left(r_{1}, r_{2}\right)=\left(115^{\circ} \mathrm{W}, 37^{\circ} \mathrm{N}\right)$. When $60^{\circ} \mathrm{E} \leq \mathrm{y} \leq 180^{\circ} \mathrm{E},\left(r_{1}, r_{2}\right)=\left(120^{\circ} \mathrm{E}, 25^{\circ} \mathrm{N}\right)$.

In the light of the destination of the delay phernmone and bandwidth phernmone, the total link phernmone can be get:

$$
\tau_{s d}=\alpha \tau_{s d_{D}}+(1-\alpha) \tau_{s d_{B}}
$$

Among them, the proportion factor $\alpha$ is used to adjust the proportional relation among phernmone. As the value of $\alpha$ increase, it means the demad of the delay is higher and lower on the contrary.

\subsection{Routing Selection of New Algorithm}

By the definition of time domain, space domain and frequency domain, the algorithm take three factors that have maximum impact to the performance of traffic into consideration. As a consequence, the link status will be perceived dynamically. Through above discussion, new routing algorithm can be divided into three processing.

a) Network initialization

The routing initialization should be set. In detail, all pheromone in the link need to be initialized in this stage to ensure initial routing selection has no influence by positive feedback of algorithm. All routes are given equal amount of pheromone and they are stored in neighboring nodes of this link. Then, the destination node will create a 'protection' packets that will be broadcast to all neighbor nodes. The packets contain a prediction area $Y$ which denotes predicted remaining amount of current node in the period of time of the future. The destination node introduces own prediction value as initialization 'protection' packet.

Then the protection information is transmitted among LEO satellites. The packets of $k_{j}$ are inserted into own information lists after the satellite $k_{i}$ receives 'protection' packet from $k_{j}$. The satellite node $k_{i}$ updates 'protection' packet on the basis of its own prediction area and then broadcast this packet to neighbor nodes. Likewise, the packets flood to source node.

b) Phernmone Dispersion

Source node will generate forward 'ant' which carries detection packets after it receives 'protection' packet which contains band measurement and delay measurement simultaneously and then it starts to detect and establish routing. Forward 'ant' selects neighbor nodes as next-hoop one in the light of state shift rule of this algorithm. 
Here, low computation complexity of calculating state shift rule should be chosen as far as possible on account of limited computing capability on the satellite. Consequently, the addition operation is replaced by multiplication to implement the combination of phernmone and heuristic information, as shown in equation 8:

The 'ant' $k$ located in node $s_{i}$ selects next satellite node $d_{j}$ according to following formula:

$$
P_{i j}=\left\{\begin{array}{c}
\frac{\xi \tau_{s d}(t)+(1-\xi) \eta_{s d}(t)}{\sum_{v \in Z_{k}(s)} \xi_{s v}(t)+(1-\xi) \eta_{s v}(t)} d \in Z_{k}(s) \text { 且 } 0<\xi<1 \\
\sum_{j \in Z_{k}(s)}^{0} P_{i j}=1
\end{array}\right.
$$

Here, $\xi$ is a system parameter, which represents the importance degree of phernmone on the communication link. $\eta_{s d}$ is called regulatory factor. It is introduced to avoid the problem that the traffic is concentrated caused by positive feedback of algorithm. If there is no regulatory factor, the state transition probability will be determined completely by phernmone on the link, which will attract more 'ant' to move toward this link. As a result, the phernmone will increase to a higher degree. The phernmone will be too concentrated if this process goes on constantly and result in the network loss the ability to adapt itself to the high dynamics. We defined the regulatory factor as:

$$
\eta_{s d}(t)=\frac{E^{g_{1}}}{B^{g_{2}}}
$$

Where $g_{1}$ and $g_{2}$ are weight coefficients of $E$ and $B$. $E$ is amount of reservation of current node and $B$ is communication traffic at time t.

$$
B=\sum_{v_{i} \in Z_{k}(s)} B_{i}
$$

$v$ is network nodes and $Z_{k}(s)$ is symbol of set of satellite nodes which have ISL with node $s$ directly.

Sub-optimal next hoop will be chosen if the neighbor node refuses to forwarding. Every time the forwarding 'ant' arrives at a node, it will judge whether the ant arrived or not. The arrived 'ant' will be cleared and no 'ant' will be generated because of the existed loop.

c) Network Dynamic Self-Learning

When forwarding 'ant' moves from node $k_{p}$ to node $k_{\text {qq }}$, relevant database table of arrived node be dynamically updated in accordance with measured band value and delay value. Above process will be repeated until $k_{q q}$ is destination node if it isn't that. Forwarding ant will be cleared automatically after arriving at destination node and backward ant be generated at once, after which the backward ant goes back along original way to the source node, carrying all information forwarding ant collected.

As the 'ants' moving around the network, the satellite link status update constantly. Phernmone updating is defined as follows:

$$
\tau(s, d)_{\text {new }}=(1-\sigma) \tau(s, d)_{\text {old }}+\sigma\left(\Delta \tau(s, d)+\quad \gamma \max _{v \in Z_{k}(s),(s, d) \in E} \tau(s, d)\right)
$$

Where $\sigma$ is phernmone volatilization factor, which determine the phernmone volatile quantity in unit time. $\gamma$ is a introduced parameter in the algorithm, which donates how the non-transmission link affect the node $v . Z_{k}(s)$ represents the set of satellite nodes between which and node $s$ exists ISL directly. $E$ is the set of communication link between nodes. The parameter increment $\Delta \tau(s, d)$ donates the parameter that is freed after ith 'ant' pass the link. The value is:

$$
\Delta \tau(s, d)= \begin{cases}\frac{Q}{L_{k}} & (s, d) \in T \\ 0 & (s, d) \notin T\end{cases}
$$

Where constant $Q$ denotes freed total phernmone during addressing process of each 'ant'. $T$ represents the search tree formed by path search. $L_{k}$ is the total path branch. Then we put equation (7) in phernmone updating equation(12) and get: 


$$
\begin{aligned}
& \tau(s, d)_{\text {new }}= \\
& (1-\sigma)\left(\alpha \tau_{\text {sddold }}+(1-\alpha) \tau_{s d_{B o I d}}\right)+\sigma\left\{\Delta \tau(s, d)+\gamma \max _{v \in Z_{k}(s),(s, d) \in E}\left[\alpha \tau_{s d_{D}}+\right.\right. \\
& \left.\left.(1-\alpha) \tau_{s d_{B}}\right]\right\}
\end{aligned}
$$

The ants move all around the satellite nodes and release phernmone constantly by satellites' releases. The phernmone on different ISLs is so disparate because of discrepancy of longitude and latitude and link status of each satellite. The state quality of each link can be distinguished effectively using above discrepancy. Obviously, more excellent links have higher probability to be the next-hoop destination node. The optimal link will be strengthened constantly and give guidance to other information to take optimal routing selection spontaneously.

d) Dynamically Transmission of Information

Assumed that each satellite's ISL is $L_{1} \ldots \ldots L_{N}$ and each ISL has amount of phernmone $\tau_{1} \ldots \ldots \tau_{N}$. All packets select link arbitrarily to transmit by probability.

$$
P_{i}=\frac{\tau_{i}}{\Sigma_{1}^{N} \tau}
$$

LEO satellite sends load state information to GEO satellite linking with it and GEO satellite maintains LEO satellite's state information to judge if it is in the state of overloaded $(\rho \geq 0.8)$. When over-loading happens, LEO satellite sends over-loading announcement to GEO satellite that will judge if it is band-sensitive traffic and can implement data transmission directly. The over-loading announcement will stop when all links are restored to normal.

\section{Result and Analysis}

Dijkstra shortest path algorithm and LPR algorithm proposed in [9] are all classical routing algorithms which are often applied to be public reference standard to evaluate performance of new models. In this paper, algorithms of Dijkstra, LPR, MS-ACO be contrasted by computer simulation under the same condition and then difference analysis of each algorithms is given.

\subsection{Simulation Parameters}

The LEO layer satellite network uses structure of like-Iridium constellation which has $66\left(6^{*} 11\right)$ satellites. GEO layer satellite network is composed by 3 geostationary satellite which have equal longitude space and distribute above equator uniformly. Other basic parameters are shown in table 2:

\section{Table 2. Main Parameters of Satellite Topology}

\begin{tabular}{ll}
\hline Parameters & Values \\
\hline LEO orbital altitude/km & 700 \\
\hline GEO orbital altitude /km & 35786 \\
\hline Inclination Angle & $86.4^{\circ}$ \\
\hline Interplane separation & $31.6^{\circ}$ \\
\hline intra-plane ISLs & 2 \\
inter-plane ISLs & 2 \\
\hline Type of ordinary queue & FIFO \\
Average packet size & $800 \mathrm{Bytes}$ \\
Maximum packet size & $2000 \mathrm{Bytes}$ \\
\hline GEO Bitrate & $25 \mathrm{Mbps}$ \\
\hline LEO Bitrate & $2.5 \mathrm{Mbps}$ \\
\hline
\end{tabular}

In addition, the phernmone adjusting parameter $\alpha$ is set as 0.8 if the traffic is delaysensitive information and is set as 0.2 otherwise. Phernmone volatilization factor $\sigma$ is set 
as $0.1, \gamma=0.25, Q=8, \quad \xi=0.4 . g_{1}, g_{2}$ is respectively set as 1.2 and 2 according to multiple simulations.

\subsection{System Performance and Analysis}

In the simulation results, packet drop rate, transmission delay and throughput are indexes to evaluate routing strategy.

Figure 5 demonstrates average transmission delay of different kind of information using each algorithm under the same condition. Compared with LPR algorithm, STC-RA algorithm has lower performance at low transmission speed when transmit delay-sensitive traffic. However, it gets constantly better performance as the transmission rate increases gradually which is superior to LPR at high speed. It is because STC-RA algorithm needs to generate phernmone transmitting around the satellite nodes when calculating, for which it consumes more processing resource than others and don't possess superiority at low speed. With the speed increases, the consumption of phernmone takes less proportion as total resource, which results getting lower growth rate of transmission delay with the speed increases. Also, the phernmone make the delay-jitter lower than LPR in LEO. To the band-sensitive information, its delay performance is better because of location factor set by hot area and forced this kind of traffic to choose idle links.

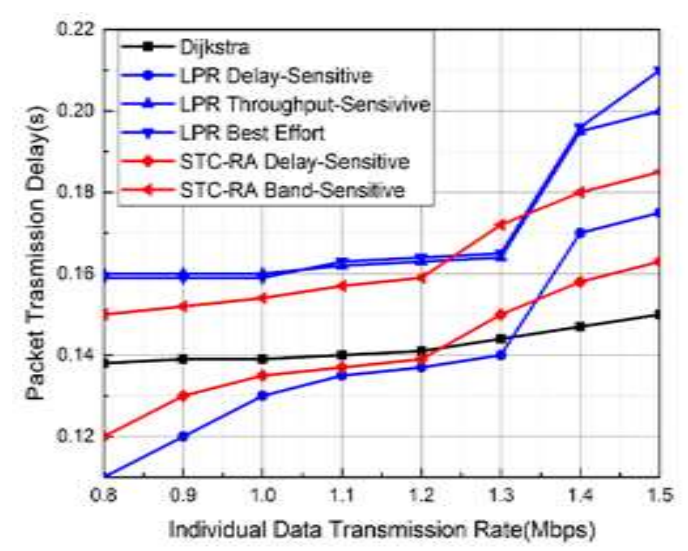

\section{Figure 5. Packet Transmission Delay}

Compared with above two algorithms, although its delay performance is better than STC-RA, the whole performance is much worse regard to its packet drop rate in figure 9.

Total packet transmission delay of three kinds of algorithms in the combination with component relationship is demonstrated in figure 6. It is manifest the performance of STC-RA and LPR are almost the same when the speed less than 1.3Mbps. The performance superiority of STC-RA is getting better with the transmission rate progressively increasing. 


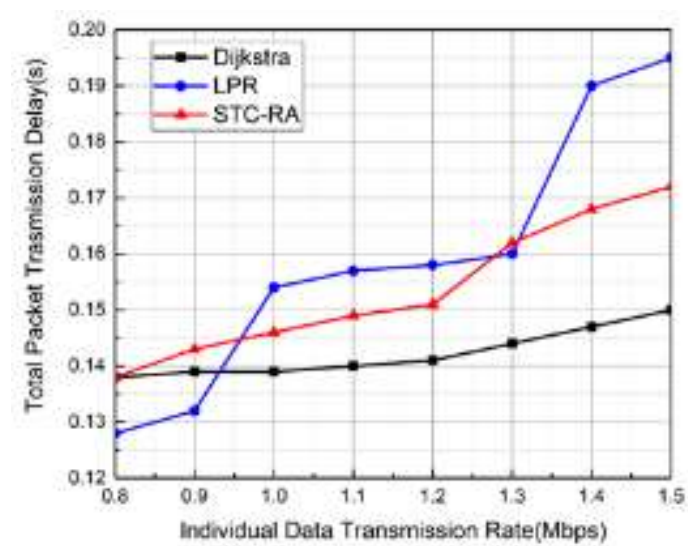

Figure 6. Total Packet Trasmission Delay

The performance of data throughput of different kind of information under same condition is shown in figure 7. From the result, no matter which kind of information it is, the performance of STC-RA is much better than Dijkstra because the Dijkstra algorithm don't allocate traffic to non-congestion effectively which lead to the phenomenon of too centralized traffic in the area of high altitude and reduction of system throughput. Compared LPR to STC-RA, the STC-RA algorithm don't have apparent advantages at the speed of more than 1.1Mbps. When the speed is over 1.1Mbps, STC-RA has better performance because the regulatory factor is used in state transfer rule whose function is to figure out the issue of too centralized of traffic caused by positive feedback of algorithm and to partly transfer traffic to idle links. Accordingly, the distribute of traffic in network is more even which caused higher throughput.

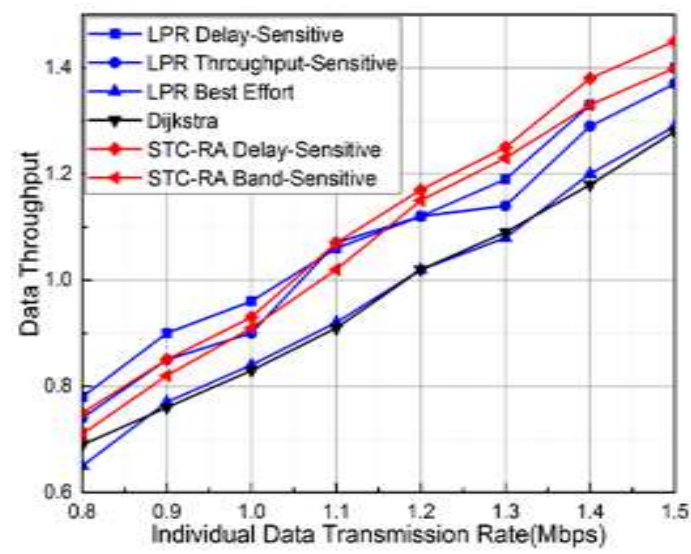

Figure 7. Data Throughput

Network average data throughput of three kinds of algorithms in the combination with component relationship is displayed in figure 8. The performance of STC-RA is better than the other two on account of its optimize design to both delay-sensitive and bandsensitive traffic in certain degree. The condition of packet drop rate is compared at the end.

The result manifests that the maximum drop rate of STC-RA is less than $0.6 \%$, almost the same as LPR and much lower than Dijkstra algorithm whose minimum drop rate is up to $1.1 \%$. The reason of getting better drop rate performance is the introduction of buffer queue delay, with which increasing, the value of phernmone decreases continuously. And then the link weight connected with it decreases to reduce the priority of this node in routing selection. Accordingly, when single node's load is too high or queuing waiting 
time is too long, the system will implement division adaptive and guarantee the traffic under stability range to avoid system drop rate increasing at high traffic speed.

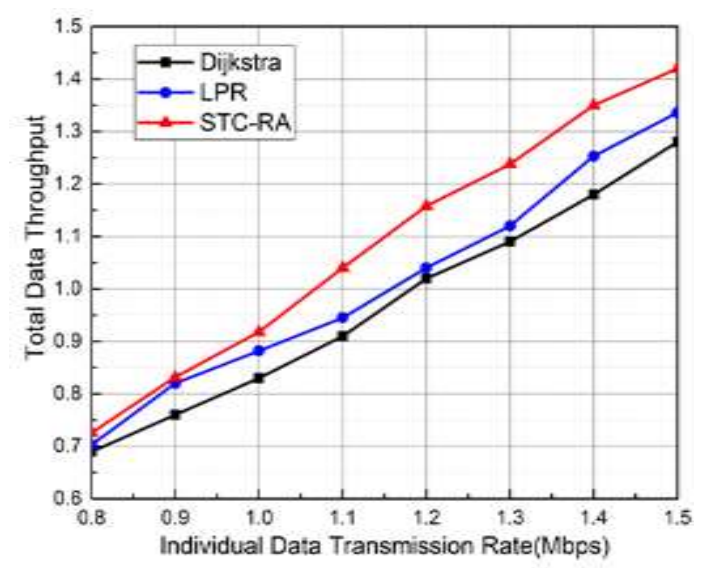

Figure 8. Total Data Throughput

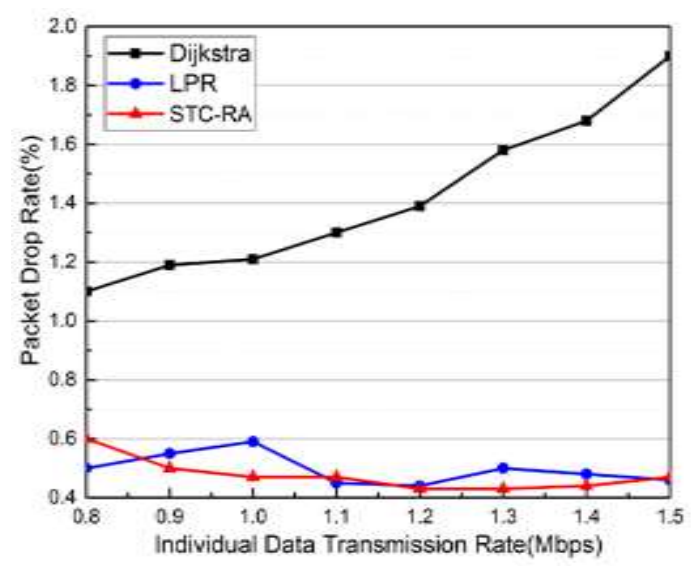

Figure 9. Packet Drop Rate

\section{Conclusion}

This paper combines fundamental of ant colony algorithm with operation and transmission characteristics of satellite network for shortages and problems of current load balancing algorithm and proposes a new one. The new algorithm not only gets excellent properties of using of ant colony algorithm in distributed network but also overcomes intrinsic problems of high dynamic and long delay in satellite network. Compared with Dijkstra algorithm, STC-RA promotes end-to-end delay and drop rate much. It also has better throughput and delay performance compared with LPR, which makes the delay decreases by $6.23 \%$ at the speed less than $1 \mathrm{Mbps}$ and the throughput increases by $6.85 \%$ in whole region. The feasibility and effectivity of STC-RA are verified by simulation results and it provides a promising method as reference for related research. 


\section{References}

[1] X. Yu, Y. Yang and Jinjun Ding, "Satellite network design method applicable to orbit determination and communication for GNSS", Software Engineering and Service Science (ICSESS), 2013 4th IEEE International Conference, vol. 25, no. 23, (2013), pp. 886-889.

[2] E. Ekici, I. F. Akyildiz and M. D. Bender, "A Distributed Routing Algorithm for Datagram Traffic in LEO Satellite Network. IEEE Transactions on Networking”, vol. 9, no. 2, (2001), pp. 137-147.

[3] M. Hussein, G. Jakllari and B. Paillassa, "On routing for extending satellite service life in LEO satellite networks", Global Communications Conference, pp. 2832-2837.

[4] L. Yong, J. Zhang and T. Zhang, "UMR: A utility-maximizing routing algorithm for delay-sensitive service in LEO satellite networks", CHINESE JOURNAL OF AERONAUTICS, vol. 2, no. 28, (2015), pp. 499-507.

[5] Z. Wu, G. Hu and J. F. Guyu, "Hop-Limited Adaptive Routing in Packet-Switched Non-Geostationary Satellite Networks", IEICE Transactions on Communications, vol. 11, no. E98B, (2015), pp. 2359-2368.

[6] D. Sirui, L. Yuanan and H. Hefei, "Load Balancing Strategies Based on a Distributed Routing in LEO Satellite Networks", Journal of University of Electronic Science and Technology of China, vol. 3, no. 43, pp. 375-380

[7] H. Nishiyama and Y. Tada, "Toward Optimized Traffic Distribution for Efficient Network Capacity Utilization in Two-Layered Satellite Networks", Vehicular Technology, vol. 3, no. 62, (2013), pp. 1303 1313.

[8] Y. Lu, Y. Zhao and S. F. Youjian, "A survivable routing protocol for two-layered LEO/MEO satellite networks", Wireless Networks, vol. 5, no. 20, (2014), pp. 871-887.

[9] L. Wang, L. Liu and X. Hu, "Load-aware and Position-aided Routing in Satellite IP Networks", International Journal of Computers Communications \& Control, vol. 1, no. 7, (2012), pp. 163-172.

[10] Karapantazis, Stylianos and Pavlidou, "QoS handover management for multimedia LEO satellite networks", Telecommunication Systems, vol. 4, no. 32, (2006), pp. 225-245.

[11] Rao,Y. Zhu and Jun, "Agent-based Multi-Service Routing for Polar-orbit LEO broadband satellite networks", AD HOC NETWORKS, vol. 13, (2014), pp. 575-597.

\section{Authors}
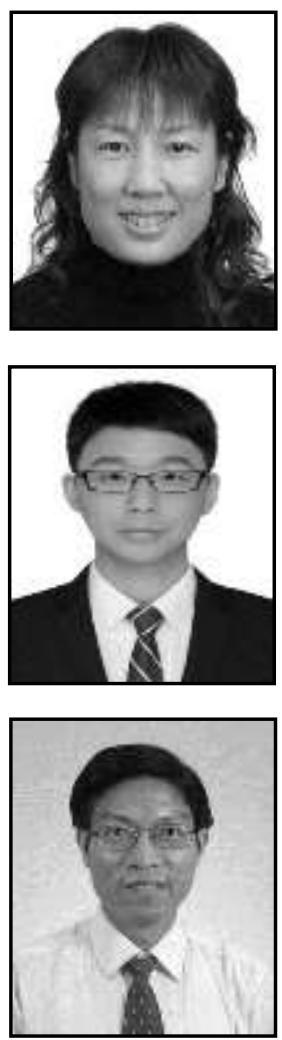

Ning Li, she is a Ph.D., associate professor at the School of Electronic Engineering, Beijing University of Posts and Telecommunications. Research Interests: Information Security, Satellite Communications.

Runtao Zhu, he is a graduate student at the school of Electronic Engineering, Beijing University of Posts and Telecommunications. Research Interests: Satellite Communications, Traffic Control.

Zhongliang Deng, he is a Ph.D., professor at the School of Electronic Engineering, Beijing University of Posts and Telecommunications. Research Interests; Satellite Navigation and Communications. 
International Journal of Multimedia and Ubiquitous Engineering

Vol. 10, No. 6 (2017) 